\title{
Initial mass function in the South-Western part of M 31
}

\author{
T. Veltchev ${ }^{1,2}$, P. Nedialkov ${ }^{1,2}$, and G. Borisov ${ }^{3}$ \\ 1 Department of Astronomy, University of Sofia, 5 James Bourchier, Sofia 1164, Bulgaria \\ e-mail: [eirene; japet] @phys.uni-sofia.bg \\ 2 Isaac Newton Institute of Chile, Bulgarian Branch, 5 James Bourchier, Sofia 1164, Bulgaria \\ ${ }^{3}$ Institute of Astronomy, Bulgarian Academy of Sciences, 72 Tsarigradsko Chaussee, Sofia 1786, Bulgaria \\ e-mail: gborisov@libra.astro.bas.bg
}

Received 9 March 2004 / Accepted 7 July 2004

\begin{abstract}
We derive an average initial mass function (IMF) for $50 \mathrm{OB}$ associations in the South-Western part of Andromeda galaxy, combining ultraviolet photometry of Hill et al. (1995, ApJ, 98, 595) and two datasets HST photometry with $J H K_{\mathrm{s}}$ photometry from the 2MASS $+I$ band photometry from Magnier et al. (1992, A\&A, 96, 379). The mean extinctions within the associations were estimated in two independent ways and the stellar masses were determined by use of probabilistic techniques. The derived slope of the IMF $\Gamma=-1.59 \pm 0.09$ is close to the universal Salpeter's law and to recent estimates for massive stars' IMF in the Galaxy and in the Magellanic Clouds.
\end{abstract}

Key words. galaxies: individual: M 31 - galaxies: stellar content - galaxies: ISM - stars: luminosity function, mass function stars: early-type - stars: supergiants

\section{Introduction}

The advances in the observational astronomy over the last two decades offered new opportunities to study the massive stellar population of Local Group galaxies beyond the Magellanic Clouds. Numerous photometric researches increased our understanding of the star-forming regions in M 31 : high-quality CCD-photometry from ground-based telescopes (Magnier et al. 1992, hereafter Mag92; Haiman et al. 1994a,b; Mochejska et al. 2001; Williams 2003), HST photometry (Hunter et al. 1996; Magnier et al. 1997; Williams \& Hodge 2001), and UV imaging with space and balloon-borne telescopes (Hill et al. 1995, hereafter H95; Pagani et al. 1999). Essential progress was made in resolving the population of dense stellar groups and complexes and the determination of their intrinsic extinction. This allowed reconstruction of the star formation history (e.g., Veltchev et al. 1999; Williams 2003) and determination of the initial mass function (IMF) in some OB associations (e.g., Haiman et al. 1994b; Hunter et al. 1996).

However, our knowledge of IMF in M 31 remains limited, especially in terms of different stellar populations. The most massive blue stars - usually used to derive the IMF - are clustered into high density groups, subject to severe image blending, even with the HST. Each of these groups spans a narrow age range, allowing for possible systematic effects. On the other hand, the red supergiants (RSGs) are more dispersed both in age and in space. An obvious problem with RSGs is the insufficient statistics in optical images, due to their faintness in comparison with the massive blue stars.
The infrared $J H K_{\mathrm{s}}$ photometry provided by the Two Micron All Sky Survey (2MASS) allows selection of RSGs in nearby galaxies, even in regions with significant extinction in the UVand optical range. Combining such selection with the available data for the OB stars, one may determine the IMF of massive (blue and red) stars in the associations.

Here we present our results for the average IMF in the South-Western part of M31, derived from the luminous stellar content of $50 \mathrm{OB}$ associations. The samples of blue and red luminous stars are described in Sect. 2; the methods of their dereddening are exposed in Sect. 3. In Sect. 4 we comment briefly the reddening in the OB associations. Section 5 is dedicated to the applied techniques for stellar mass estimation. The results for the IMF are reported in Sect. 6 and discussed in Sect. 7.

\section{Stellar samples}

Ultraviolet photometry in bands centered at $\lambda_{\text {eff }}=2490 \AA$ and $\lambda_{\text {eff }}=1520 \AA$ was obtained for 611 stars in 59 van den Bergh (1964) associations by H95. We retain further the traditional term "associations" although these groups were resolved later into real OB associations with typical size of 80 pc (Efremov et al. 1987). H95 complemented the UV photometry by CCD B band magnitudes, partly from the catalog of Mag92. After extinction correction for each association, the color-magnitude diagram (CMD) $m_{2490}$ vs. $\left(m_{2490}-B\right)$ clearly shows that the sample contains massive OB stars $\left(\mathcal{M} \geq 10 \mathcal{M}_{\odot}\right)$ and a "plume" of foreground stars with a positive color value (see Fig. 18 


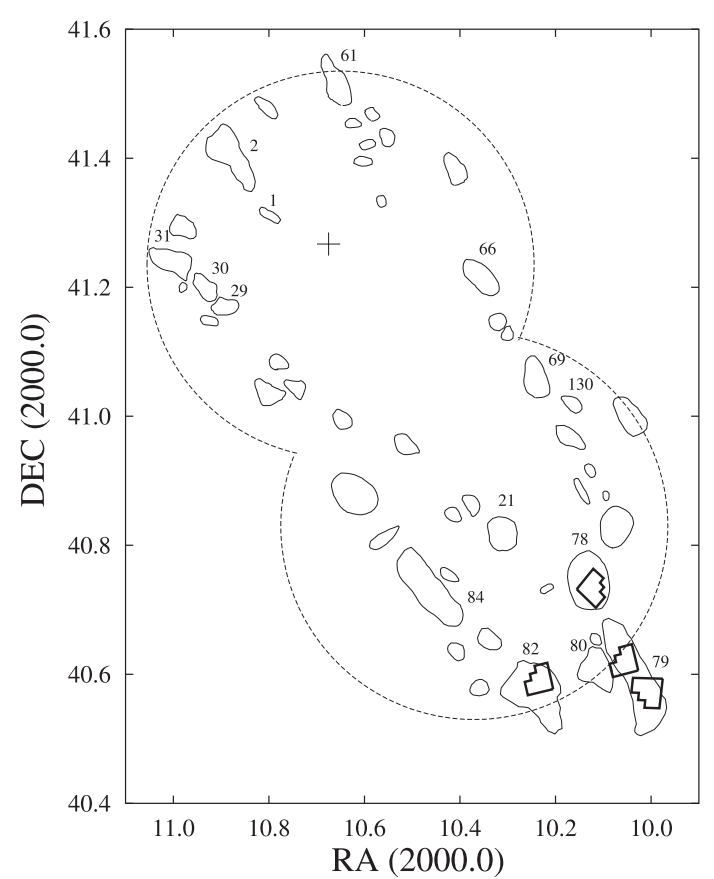

Fig. 1. Map of the selected OB associations. The area covered in the study of H95 is outlined (dashed line) and the van den Bergh (1964) numbers of the richest OB associations are given. Four WFPC2 fields with HST photometry of Hunter et al. (1996) and Williams (2002) are plotted with thick lines (see text).

in H95). Removing the foreground and several other objects, identified as globulars, we retained for further consideration 459 blue stars in 46 associations (Fig. 1).

Next, we selected RSG candidates with $\left(J-K_{\mathrm{s}}\right)>1.1$ from the 2MASS catalog within these 46 plus 4 additional SouthWestern associations in the area covered in H95 (see Table 1). The foreground dwarf contamination is negligible, as indicated by a comparison of the CMD with that of a nearby field of equal area, located outside the disk of M31. I-band magnitudes of 208 RSG candidates, necessary for the dereddening procedure, were obtained from Mag92. Table 1 lists the number of sample stars in each OB association.

Eventually, HST photometry in van den Bergh associations OB78 (Hunter et al. 1996), OB79 and OB82 (Williams 2002) was used to obtain additional reference points as we derive the IMF (see Sect. 6). Two of the WFPC2 fields fall within and the others - outside the area, covered in the study of H95 (see Fig. 1).

\section{Dereddening procedures}

\subsection{Individual dereddening of RSG candidates}

The color-color diagram $\left(I-K_{\mathrm{S}}\right)$ vs. $\left(J-K_{\mathrm{S}}\right)$ allows to derive the individual reddening of RSG candidates using the classical Q-method. The red ends of the stellar evolutionary tracks for masses 12-20 $\mathcal{M}_{\odot}$ and metallicities $Z=0.02-0.05$ (Bressan et al. 1992; Fagotto et al. 1994) coincide within the typical photometric error of the sample (Fig. 2). These tracks determine a zero-extinction line, crossed under significant angle by the reddening vector. We assume that the extinction of RSGs is mainly
Table 1. Sample parameters. The identification of the selected OB associations according to van den Bergh (1964), the area, and the number of OB stars from $\mathrm{H} 95$ and red supergiants are given. The parenthesis in Col. 4 contain the number of RSG candidates for which reddening correction was possible.

\begin{tabular}{|c|c|c|c|}
\hline $\begin{array}{l}\text { Assoc. } \\
\text { ID }\end{array}$ & $\begin{array}{c}\text { Area } \\
{\left[\operatorname{arcmin}^{2}\right]}\end{array}$ & $\begin{array}{c}\mathrm{OB} \\
\text { stars }\end{array}$ & $\begin{array}{c}\text { RSG } \\
\text { candidates }\end{array}$ \\
\hline 1 & 1.16 & 9 & - \\
\hline 2 & 10.33 & 32 & $2(2)$ \\
\hline 12 & 1.78 & 5 & 1 (1) \\
\hline 13 & 0.91 & 2 & - \\
\hline 14 & 0.77 & 3 & 1 (1) \\
\hline 15 & 0.81 & 4 & $2(2)$ \\
\hline 16 & 0.78 & 2 & - \\
\hline 21 & 7.30 & 34 & 8 (7) \\
\hline 22 & 2.16 & 4 & 3 (3) \\
\hline 23 & 1.56 & 1 & $2(2)$ \\
\hline 24 & 3.13 & 5 & 4 (4) \\
\hline 25 & 2.44 & 2 & 3 (3) \\
\hline 26 & 2.29 & 2 & 1 (1) \\
\hline 27 & 1.98 & 2 & 1 (1) \\
\hline 29 & 3.14 & 13 & $5 \quad(5)$ \\
\hline 30 & 3.77 & 10 & 12 (12) \\
\hline 31 & 7.34 & 14 & 20 (18) \\
\hline 35 & 3.96 & 6 & $2(2)$ \\
\hline 61 & 7.69 & - & 22 (19) \\
\hline 62 & 1.18 & - & 4 (4) \\
\hline 63 & 1.86 & 3 & 2 (2) \\
\hline 66 & 6.98 & 5 & 11 \\
\hline 67 & 2.08 & 6 & 3 (3) \\
\hline 68 & 1.13 & 3 & 1 (1) \\
\hline 69 & 7.00 & 15 & 3 (3) \\
\hline 70 & 3.75 & 8 & $1(-)$ \\
\hline 71 & 1.47 & 4 & 1 (1) \\
\hline 72 & 9.12 & 8 & 2 (2) \\
\hline 74 & 0.73 & 3 & 4 (4) \\
\hline 75 & 0.91 & 4 & 1 (1) \\
\hline 76 & 1.43 & - & 2 (2) \\
\hline 77 & 3.84 & 6 & 3 (1) \\
\hline 78 & 17.15 & 105 & 27 (27) \\
\hline 79 & 31.68 & 6 & $8 \quad(5)$ \\
\hline 80 & 11.20 & 23 & 19 (19) \\
\hline 82 & 28.80 & 29 & 23 (19) \\
\hline 83 & 3.23 & 9 & 3 (3) \\
\hline 84 & 20.61 & 17 & $10 \quad(8)$ \\
\hline 85 & 2.79 & 7 & 1 (1) \\
\hline 86 & 12.62 & 6 & $2(2)$ \\
\hline 89 & 4.37 & - & 1 (1) \\
\hline 90 & 1.19 & 2 & - \\
\hline 91 & 0.51 & 7 & - \\
\hline 117 & 4.27 & 1 & 1 (1) \\
\hline 121 & 7.16 & 2 & $4 \quad(3)$ \\
\hline 130 & 1.98 & 10 & - \\
\hline 131 & 0.94 & 3 & - \\
\hline 132 & 0.41 & 4 & - \\
\hline 144 & 1.19 & 7 & - \\
\hline 145 & 1.74 & 6 & 1 (1) \\
\hline Sum & 241.95 & 459 & $227(208)$ \\
\hline
\end{tabular}




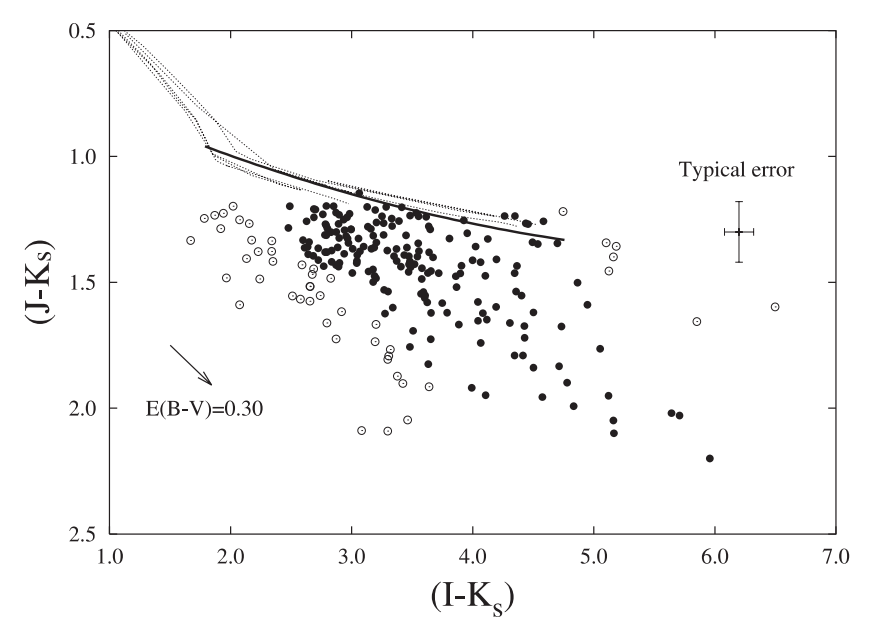

Fig. 2. The sample of RSG candidates on color-color diagram. The successfuly dereddened objects are plotted with filled circles. Some evolutionary tracks (dotted lines), the fitting zero-absorption line (thick line) and the reddening vector are drawn.

affected by the diffuse component of the interstellar medium and adopt Galactic extinction curve, characterized by $R_{V}=3.1$ (Fitzpatrick 1999).

There are two distinctive groups (marked with open circles) outside the limiting lines for possible dereddening. The group on the left can be RSGs of class M with underestimated color (due to photometric errors) and/or their shortly living K counterparts. The few extremely red objects seem to be blended or non-stellar images.

The successfully dereddened objects are RSGs of class M. The errors of the true color were calculated by applying the Q-method to 8 different sets of color values, corresponding to the corners and size-centers of the photometric error box. The final sample, used to derive IMF and as a reference point for the reddening within the association (see Sect. 4), contains objects with at least 4 (out of 8) successful "error-box dereddenings".

\subsection{Derivation of mean color excess and UV extinction}

H95 estimated the mean optical excess $E_{\mathrm{M} 31}(B-V)$ and the normalized UV extinction $R_{2490, \mathrm{M} 31}=A_{2490, \mathrm{M} 31} / E_{\mathrm{M} 31}(B-V)$ within each association as the corrections that maximize the correlation of the corrected stellar magnitudes with the luminosity class (I, III and V) sequences on the CMD (see Fig. 16 in H95). The excesses vary from 0.0 to $0.35 \mathrm{mag}$ while in almost all cases $R_{2490, \mathrm{M} 31}=6.60$, corresponding to $R_{V, \mathrm{M} 31}=$ 3.1. Adding constant foreground reddening inside the Milky Way in the direction towards M $31 E_{\mathrm{MW}}(B-V)=0.08$ (Burstein \& Heiles 1984), we obtained for the total excess, averaged over the studied associations: $\left\langle E_{\text {tot }}(B-V)\right\rangle=0.25$. The CMD of our sample of 459 OB stars, dereddened with values of $R_{2490, \mathrm{M} 31}$ and $E_{\mathrm{M} 31}(B-V)$ from Table 3 in H95, is plotted in Fig. 3. The absolute magnitudes are calculated with distance modulus of 24.47 mag (Stanek \& Garnavich 1998). The sample points occupy horizontally extended locus. Only part of them fall within the Main-sequence (MS) strip, as outlined by

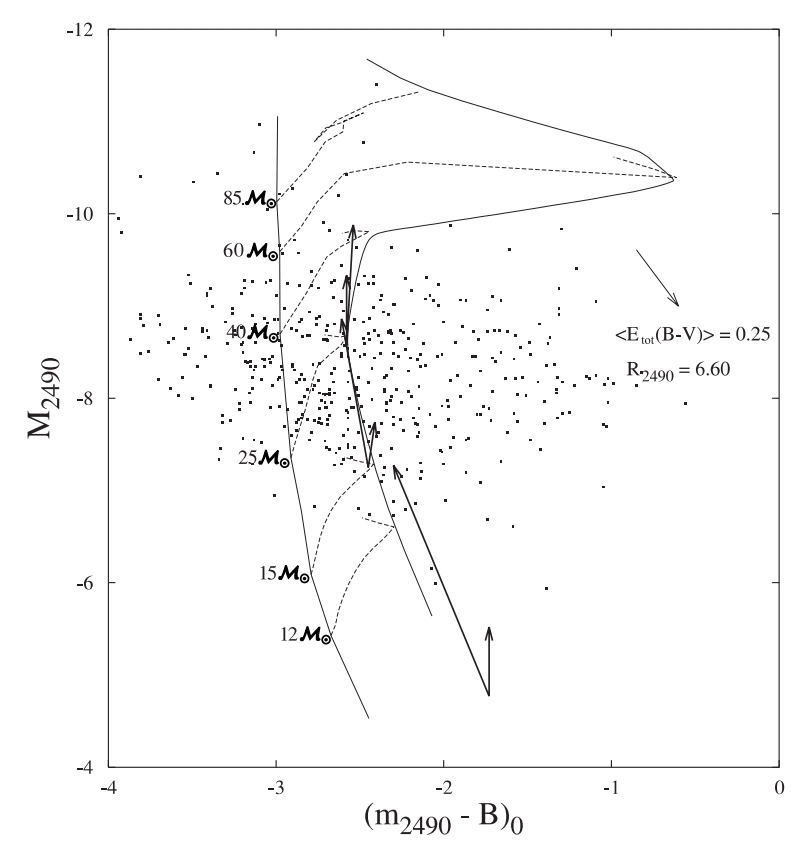

Fig. 3. Color-magnitude diagram of the OB stars sample, dereddened with values of $R_{2490, \mathrm{M} 31}$ and $E_{\mathrm{M} 31}(B-V)$ from H95. The MS strip boundaries (solid) and the MS sections of the Geneva evolutionary tracks (dashed) are plotted. The reddening vector is drawn; the thick arrows indicate the shift of MS stars due to possible image blending with other massive MS stars.

Geneva evolutionary tracks (Schaller et al. 1992), computed in terms of color and magnitude by use of model atmospheres of Kurucz (1992). The objects with $\left(m_{2490}-B\right)_{0}<-3$ can be MS stars overcorrected for extinction, blended images of MS stars in dense association regions or post-MS stars, e.g. Wolf-Rayet stars (WR). Their number is 61 while there are 50 dereddened RSG candidates of the corresponding mass range $\mathcal{M}>15 \mathcal{M}_{\odot}$ $\left(M_{V}<-5\right.$.). The number ratio is comparable with the observed RSG/WR $=1.35$ in 7 associations in M 31 (Massey \& Johnson 1998).

The bulk of objects on the right of MS strip are not postMS stars - they fall into the Hertzsprung gap, corresponding to extremely short evolutionary lifetimes. The possible explanations of their positions can be: i) image blending of OB stars and RSGs, leading to redder colors; ii) underestimated (individual) extinctions or; iii) incorrect $R_{2490, \text { M } 31}$ estimates. The explanation i) does not seem plausible for two reasons. First, the RSG evolutionary lifetime is about an order shorter, - and hence their spatial density within the associations substantially less, - than those of OB stars with similar masses. Therefore image blending of a MS star with other MS star(s) is much more likely than with an RSG. On the other hand, the UV color range of high-mass MS stars is narrow and their blending affects mostly the luminosity than the color of the resulting object (Fig. 3). The possibility ii) can be verified by adopting the typical values of $R_{2490, \text { M } 31}$ given in H95. The slope of the reddening vector on the CMD $\mathrm{M}_{2490}$ vs. $\left(m_{2490}-B\right)_{0}$ increases with $E(B-V)$ and thus the objects with large reddening would be stars of very high mass - around and beyond the upper observational limit. In the best case, most of them fall within the 


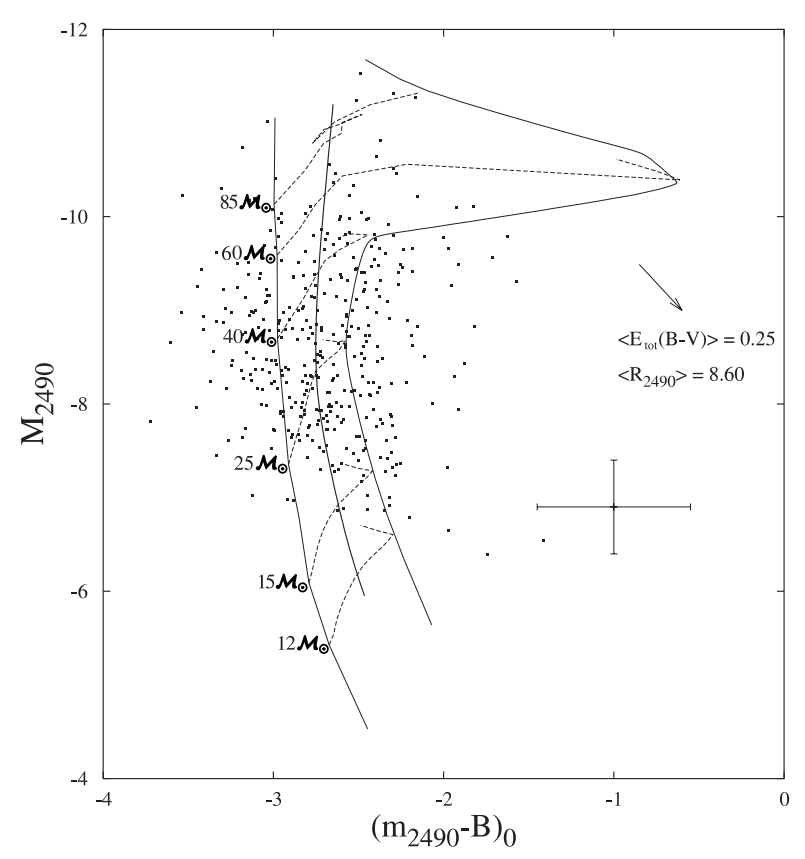

Fig. 4. Color-magnitude diagram of a subsample of 377 OB stars, dereddened with values of $R_{2490, \mathrm{M} 31}$ and $E_{\mathrm{M} 31}(B-V)$ for each association, obtained through our minimization procedure (see text). The central MS line (thick solid) and the typical error box are drawn. The other symbols are the same as in Fig. 3.

mass range $60-80 \mathcal{M}_{\odot}$ and surpass in number the stars of lower masses.

The veracity of the explanation iii) is demonstrated through a procedure for simultaneous derivation of the mean color excess and normalized UV extinction on the CMD of each assosiation. The initial step was to eliminate the foreground UV extinction from the Milky Way - all stars in the sample were dereddened with constant $A_{2490, \mathrm{MW}}=0.62(\mathrm{H} 95)$ and $R_{V, \mathrm{MW}}=$ $3.1\left(R_{2490, \mathrm{MW}}=6.60\right)$. Next, we selected only objects with apparently small intrinsic extinctions, i.e. located within $<1 \sigma$ from the MS strip boundaries on the association's CMD. By use of this subsample we performed two-parameter minimization of the rms deviation from a central MS line that connects points of mean color and luminosity for a given stellar mass (see Fig. 4). The variation limits of the parameters $E_{\mathrm{M} 31}(B-V)$ and $R_{2490, \text { M } 31}$ were $(0,0.25)$ and $(5.0,10.0)$ respectively, in accord with the results of Williams (2003; see Fig. 2 there) and the extinction curve of Fitzpatrick (1999). To estimate the uncertainties of the derived mean $E_{\text {tot }}(B-V)$ and $R_{2490}$ toward a studied association, we deredden eventually each subsample star in two alternative ways: a) fixing $E_{\text {tot }}(B-V)$ and calculating $R_{i}$ that minimizes the distance from the $i$ th star to the central MS line; b) fixing $R_{2490}$ and calculating star's individual excess $E_{i}(B-V)$. Thus the mean square errors of our procedure are:

$$
\begin{aligned}
& \sigma_{R}=\sqrt{\frac{1}{k(k-1)} \sum_{i=1}^{k}\left(R_{i}-R_{2490}\right)^{2}} \\
& \sigma_{E(B-V)}=\sqrt{\frac{1}{k(k-1)} \sum_{i=1}^{k}\left(E_{i}(B-V)-E_{\mathrm{tot}}(B-V)\right)^{2}}
\end{aligned}
$$

Table 2. UV extinction and reddening of individual OB associations. The number of OB stars $n$ in each association and the number of stars $k$ used to derive $\left(R_{2490}, E(B-V)\right)$ are given in Col. 2. The normalized UV extinctions $R_{2490}$ and their errors are listed in Col. 3. Mean color excesses from OB stars (Col. 4) and from the dereddened RSGs (Col. 5) are compared with H95 (Col. 6). The Milky Way extinction is included.

\begin{tabular}{rrrccc}
\hline \hline Assoc. & $n(\mathrm{k})$ & $R_{2490}$ & \multicolumn{3}{c}{$E_{\text {tot }}(B-V)$} \\
& & & OB stars & RSGs & H95 \\
\hline 2 & $32(22)$ & $9.30 \pm 0.37$ & $0.14 \pm 0.01$ & 0.40 & 0.18 \\
12 & $5(4)$ & $5.05 \pm 2.86$ & $0.09 \pm 0.10$ & 0.34 & 0.16 \\
14 & $3(3)$ & $9.85 \pm 0.11$ & $0.32 \pm 0.06$ & 0.40 & 0.38 \\
21 & $34(24)$ & $9.95 \pm 0.35$ & $0.17 \pm 0.01$ & 0.66 & 0.17 \\
24 & $5(3)$ & $8.10 \pm 0.46$ & $0.33 \pm 0.04$ & 0.48 & 0.28 \\
29 & $13(9)$ & $9.15 \pm 0.39$ & $0.30 \pm 0.04$ & 0.46 & 0.16 \\
30 & $10(4)$ & $6.00 \pm 0.92$ & $0.21 \pm 0.13$ & 0.57 & 0.16 \\
31 & $14(10)$ & $7.20 \pm 0.78$ & $0.26 \pm 0.11$ & 0.61 & 0.13 \\
66 & $5(2)$ & $7.20 \pm 1.98$ & $0.20 \pm 0.11$ & 0.31 & 0.28 \\
67 & $6(4)$ & $7.70 \pm 1.09$ & $0.30 \pm 0.09$ & 0.36 & 0.15 \\
69 & $15(11)$ & $8.20 \pm 0.54$ & $0.19 \pm 0.02$ & 0.32 & 0.23 \\
72 & $8(6)$ & $9.90 \pm 0.83$ & $0.18 \pm 0.02$ & 0.32 & 0.28 \\
74 & $3(3)$ & $10.00 \pm 0.00$ & $0.32 \pm 0.19$ & 0.45 & 0.32 \\
75 & $4(3)$ & $9.25 \pm 0.51$ & $0.28 \pm 0.05$ & 0.48 & 0.32 \\
78 & $105(53)$ & $7.85 \pm 0.27$ & $0.17 \pm 0.01$ & 0.29 & 0.15 \\
80 & $23(16)$ & $9.25 \pm 0.45$ & $0.15 \pm 0.01$ & 0.64 & 0.16 \\
82 & $29(16)$ & $9.70 \pm 0.22$ & $0.27 \pm 0.02$ & 0.43 & 0.32 \\
83 & $9(7)$ & $9.95 \pm 0.39$ & $0.21 \pm 0.02$ & 0.49 & 0.32 \\
84 & $17(8)$ & $7.80 \pm 0.57$ & $0.31 \pm 0.05$ & 0.86 & 0.32 \\
85 & $7(5)$ & $7.75 \pm 0.71$ & $0.30 \pm 0.05$ & 0.56 & 0.20 \\
86 & $6(4)$ & $8.30 \pm 0.57$ & $0.30 \pm 0.04$ & 0.09 & 0.42 \\
145 & $6(4)$ & $6.80 \pm 0.81$ & $0.29 \pm 0.09$ & 0.69 & 0.20 \\
\hline & & & & &
\end{tabular}

where $k$ is the number of the subsample stars in the association (see Col. 2 in Table 2). As seen from Col. 3 in Table 2, the obtained values of $R_{2490}$ are systematically higher than the estimates of H95 and lead to flatter slopes of the reddening vector.

\section{Reddening in the $\mathrm{OB}$ associations}

Other results of the dereddening procedures are given in Table 2, Cols. 4-6. The obtained mean color excesses (Col. 4) are close to the values in $\mathrm{H} 95$ (Col. 6) while the average values of the independently derived reddenings of RSGs (Col. 5), are higher by a factor of $\sim 2$. The latter result can be explained with the spatial distribution of the detected RSGs. Their statistics is less influenced by the local optical depth since the 2MASS infrared photometry allows their detection even in case of heavy reddening. Assuming homogeneous distribution of interstellar medium (ISM) in an association, the color excess should correlate with star's location on the line of sight: the "deeper" a star is immersed, the larger the reddening. Thus the mean reddening of the RSGs in a given association is representative for more obscured sightlines.

We use the average excess of RSGs to deal with the significantly reddened $\mathrm{OB}$ stars that were excluded from the dereddening procedure and still remain in the Hertzsprung gap $\left(\left(m_{2490}-B\right)_{0}>-2.2\right)$ after dereddening with the obtained $E(B-V)(C o l .4)$. For most associations, the number of successfully dereddened RSGs is small and their excesses are biased 


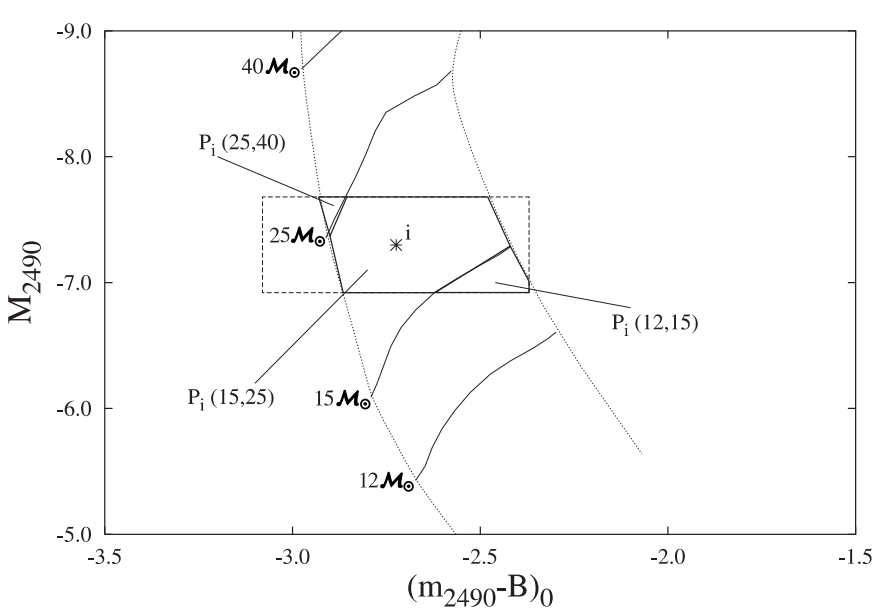

Fig. 5. Constructing the IMF. The errorbox of a star is shown with dashed lines while the fractions of the error box that overlap with the various mass bins are outlined with solid line. The other symbols are the same as in Fig. 3.

toward higher values due to requirements of the procedure (see Sect. 3.1). Therefore we attributed to significantly reddened OB stars excesses that are arithmetic means from the data in Cols. 4 and 5.

\section{Techniques for derivation of IMF}

Eventually, we select a subsample of 377 dereddened OB stars, whose individual error boxes overlap with the MS strip (Fig. 4). This subsample was used for derivation of the average IMF.

\subsection{OB stars}

Usually, the IMF is derived by counting stars between the evolutionary tracks for different initial masses in a CMD. However, this technique can not be applied to our data set because of the significant uncertainties in magnitude and color, resulting from the errors of $R_{2490}$ and $E(B-V)$ (see Fig. 4).

This problem prompted us to develop a probabilistic technique for mass estimates of individual stars. The probability that the mass of the $i$ th star falls within the interval $\left[\mathcal{M}_{1}, \mathcal{M}_{2}\right]$ is proportional to the fraction $P_{i}(1,2)$ of its error box area that overlaps with the MS strip between the tracks for $\mathcal{M}_{1}$ and $\mathcal{M}_{2}$ (Fig. 5). Therefore, we examine the error boxes of individual stars, and distribute the area of each error box over the mass bins in the range $>15 \mathcal{M}_{\odot}$ (see next section). The method produces a quasi-continuous IMF. The error box parts, that do not overlap with the MS strip, were neglected.

\subsection{RSG candidates}

As seen from the diagram $M_{K}$ vs. $\left(I-K_{\mathrm{s}}\right)_{0}$ (Fig. 6a), the masses of the derredened RSG candidates are in the range 10-25 $\mathcal{M}_{\odot}$. The evolutionary tracks have a complex shape (loops) and depend essentially on metallicity $Z$. Taking into account the uncertainties in colors and magnitudes, the standard approach to derive IMF is not appropriate for the RSG candidates. We estimate the mass of each star through
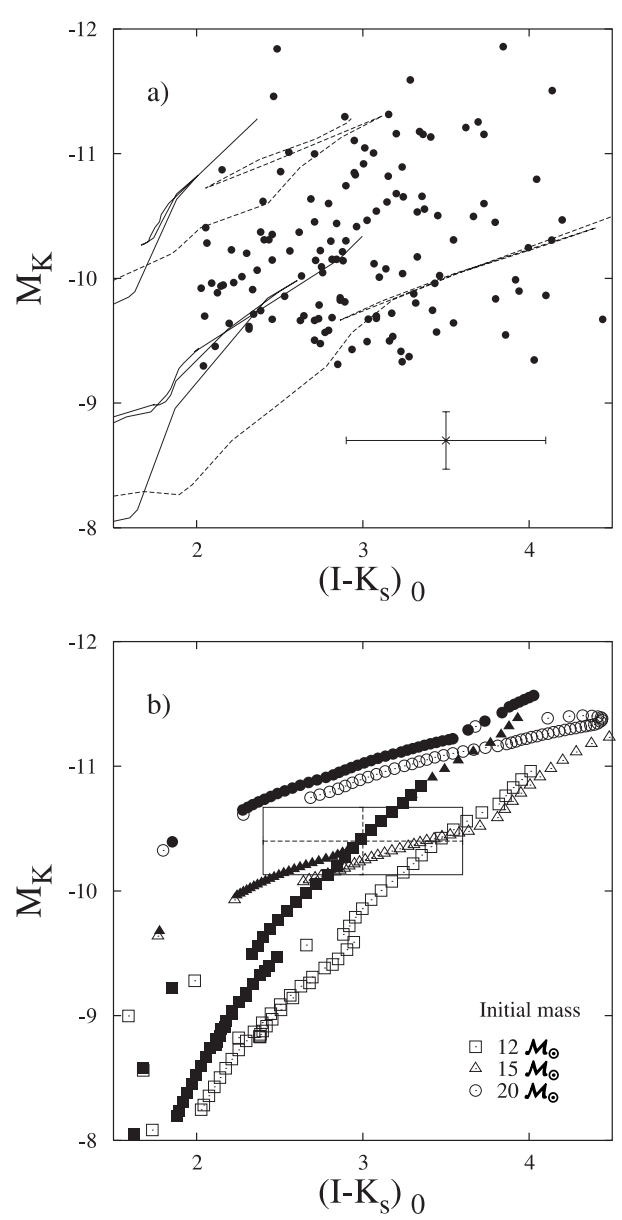

Fig. 6. IMF from the red supergiants: a) CMD of all sample stars; the typical error and the evolutionary tracks for 12 and $20 \mathcal{M}_{\odot}$ and for $Z=0.02$ (solid) and $Z=0.05$ (dashed) are shown; b) illustration of the "bullets-method" for mass determination of individual stars (see Sect. 5.2). The approximations of Hurley et al. (2000) for $Z=0.020$ (filled circles) and $Z=0.024$ (open circles) are shown.

a "bullets-method". The analytic formulae of Hurley et al. (2000) allow calculation of approximate tracks for arbitrary masses and metallicities with fixed, constant time step. The probability that the initial mass and the metallicity of a star are $\mathcal{M}_{0}$ and $Z_{0}$ respectively, is proportional to the number of points ("bullets") $N_{i}$ of corresponding evolutionary track $\left(\mathcal{M}_{i}, Z_{i}\right)$ within the error box related to that star (Fig. 6b).

The limits on metallicity were selected based on the $[\mathrm{O} / \mathrm{H}]$ gradient in M $31\left(-0.06 \mathrm{dex} \mathrm{kpc}^{-1}\right)$, derived by Galarza et al. (1999) in their study of $\mathrm{H}_{\text {II }}$ regions and supernova remnants. We converted their results from $[\mathrm{O} / \mathrm{H}]$ to $Z$, adopting relative element abundances of Anders \& Grevesse (1989). The metallicity range of the probed South-Western region of Andromeda is then $0.016 \lesssim Z \lesssim 0.024$. Therefore, we used evolutionary tracks with $Z=0.016,0.020$ and 0.024 , for initial masses 7-26 $\mathcal{M}_{\odot}$, binned in steps of $\Delta \mathcal{M}=0.5 \mathcal{M}_{\odot}$. The derived initial stellar mass and its error are given by:

$\mathcal{M}=\sum_{i} \frac{N_{i} \mathcal{M}_{i}}{N} \quad \sigma_{\mathcal{M}}=\sqrt{\frac{\sum_{i} N_{i} \mathcal{M}_{i}{ }^{2}-\mathcal{M}^{2}}{N}}$ 
Table 3. High-mass IMF. The photometrically covered area in the rectified plane, the mean evolutionary lifetimes for the corresponding mass range, the number of stars falling into each bin $N_{*}$ and the derived IMF $\log \xi$ are given. Values of $N_{*}$ for OB79 and OB82 were calculated by use of our probabilistic technique (see Sect. 5.1). The mass bins $15-25 \mathcal{M}_{\odot}$ and 25-40 $\mathcal{M}_{\odot}$ (H95) were not used to derive the IMF slope.

\begin{tabular}{|c|c|c|c|c|}
\hline $\begin{array}{c}\text { Mass range } \\
{\left[\mathcal{M}_{\odot}\right]}\end{array}$ & $\begin{array}{c}\text { Total area } \\
{\left[\mathrm{kpc}^{2}\right]}\end{array}$ & $\begin{array}{c}\text { Mean lifetime } \\
{[\mathrm{Myr}]}\end{array}$ & $\overline{N_{*}}$ & $\log \xi$ \\
\hline & OB stars (H95) & & & \\
\hline $15-25$ & 55.80 & 9.00 & 55 & -0.31 \\
\hline $25-40$ & 55.80 & 5.36 & 56 & -0.03 \\
\hline $40-60$ & 55.80 & 3.88 & 37 & -0.01 \\
\hline $60-85$ & 55.80 & 3.13 & 14 & -0.29 \\
\hline \multirow[t]{2}{*}{$85-120$} & 55.80 & 2.69 & 5 & -0.65 \\
\hline & OB stars (HST) & & & \\
\hline $15-25$ (OB78) & 1.15 & 9.00 & 55 & -0.31 \\
\hline 7-9 (OB79) & 2.09 & 34.80 & $108.16^{*}$ & 1.14 \\
\hline \multirow[t]{2}{*}{ 7-9 (OB82) } & 1.12 & 34.00 & $80.63^{*}$ & 1.28 \\
\hline & RSGs (2MASS+Mag92) & & & \\
\hline $13-17$ & 53.00 & 1.55 & 84 & 0.94 \\
\hline $17-21$ & 53.00 & 1.10 & 22 & 0.61 \\
\hline $21-25$ & 53.00 & 0.87 & 10 & 0.46 \\
\hline
\end{tabular}

where $N_{i}$ is the number of "bullets" with mass $\mathcal{M}_{i}$, and $N$ is the total number of "bullets" in the error box, associated with a given star.

\section{Average IMF in the associations}

While the asumption of coeval star formation can be adopted for a single OB association, it is invalid for our sample because it includes various associations with different ages, formed during incoherent episodes of star formation. In some cases, several incoherent starbursts can occur even within a single van den Bergh "association" (see e.g., Veltchev et al. 1999). Considering the large area covered by our sample we assumed continuous and steady-state star formation over the past 15-17 Myr. Then the average star formation rate over all associations corresponds to an average IMF, i.e. the average number of stars $N_{*}$ born in the associations per year per area per unit logarithmic mass interval $\xi(\log \mathcal{M})$. (This definition is analogous to the "field stars IMF" as an average characteristic of more modest star forming events; see Massey 2002.) Hereafter, we will refer to the average IMF in the associations simply by the term IMF. The total projected surface areas of the associations are $55.8 \mathrm{kpc}^{2}$ (OB stars) and $53 \mathrm{kpc}^{2}$ (RSGs), adopting inclination angle of $\mathrm{M} 31 i=77^{\circ}$.

A brief inspection of the derived $N_{*}$ and $\log \xi$ (Table 3) reveals incompleteness in the mass bins $15-25 \mathcal{M}_{\odot}$ and even 25-40 $\mathcal{M}_{\odot}$. To overcome the problem, we use two datasets with deeper HST photometry: a) OB stars in the giant complex NGC 206 (Hunter et al. 1996; see Fig. 1), dereddened individually by Veltchev et al. (1999); b) OB stars in van den Bergh associations OB79 and OB82 (Fig. 1), dereddened by use of mean extinction estimates (Williams 2002). In case (a) the

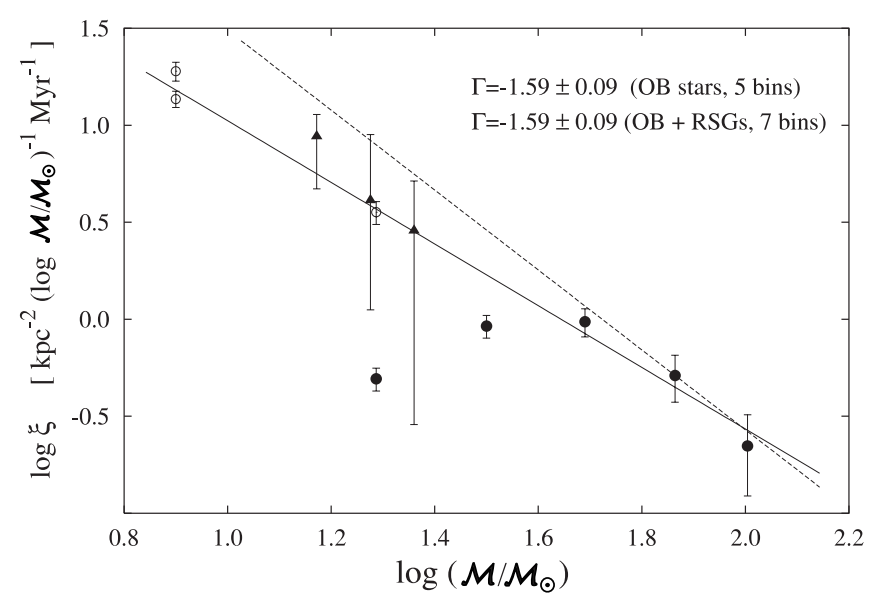

Fig. 7. The derived IMF for OB stars (filled circles) and RSGs (triangles). The values in the mass bins 7-9 $\mathcal{M}_{\odot}$ and 15-25 $\mathcal{M}_{\odot}$ obtained on the base of HST photometry (see text) are plotted with open circles. The normalized IMF, derived by Haiman et al. (1994), is shown for comparison (dotted line).

completeness of the $15-25 \mathcal{M}_{\odot}$ mass bin is $100 \%$ while the images of more luminous objects were saturated. Photometric and deredenning errors are minor and the number of stars between the tracks for 15 and $25 \mathcal{M}_{\odot}$ on CMD can be determined directly. In case (b) the upper brightness limit is $\sim 18^{m}-19^{m}$ and the highest complete mass bin is 7-9 $\mathcal{M}_{\odot}$. Adopting the errors of $A_{V}$ and $E(B-V)$ as calculated by the author (B. Williams), we obtain $(\log \xi)_{\mathrm{OB} 79}$ and $(\log \xi)_{\mathrm{OB} 82}$ in $7-9 \mathcal{M}_{\odot}$ mass bin through our probabilistic technique (see Sect. 5.1). The results are summarized in Table 3. Assuming that all associations in our sample are subjected to similar incompleteness effects, we brought up to five the number of bins over which one could derive OB stars' IMF. The slope of the IMF weighted fit is $\Gamma=-1.59 \pm 0.09$, assuming Poisson statistics $1 / N^{1 / 2}$ (Fig. 7).

The including of RSGs for derivation of high-mass IMF may raise the question whether they actually belong to the $\mathrm{OB}$ associations as a result of the same star formation event. Massey et al. (1995) distinguish "field stars" from association members, taking into account the association sizes and the mean velocities of newly born stars relative to the parental cloud (see Sect. 2.6 there). Adopting their criterion, with correction for the inclination angle of M 31 and the typical ages of this population, we can consider as association members RGSs within up to 3 arcsec from the association boundaries. Moreover, as we already pointed out (Sect. 2), most van den Bergh (1964) objects are actually stellar complexes, with considerably larger size in comparison with genuine associations. To address the question in terms of statistics, the RSGs' lifetime is of the same order like the lifetime of OB stars in the upper mass bin considered $\left(85-120 \mathcal{M}_{\odot}\right)$. Thus the including of these objects for derivation of high-mass IMF is justified. In fact, it does not change the result: $\Gamma=-1.59 \pm 0.10$ (Fig. 7). The analysis of the derived RSG masses shows lower completeness limit $\sim 13 \mathcal{M}_{\odot}$ and an upper limit of $\sim 25 \mathcal{M}_{\odot}$. The errors are typically $1<\sigma_{\mathcal{M}}<2$. We divided the mass range into 3 bins with size $4 \mathcal{M}_{\odot}$. The objects with larger $\sigma_{\mathcal{M}}$, 
that can "migrate" in the neighbor bins, determine the errors of the derived IMF.

\section{Discussion}

Our result is almost identical to the Salpeter's law (1955) and hints again at the universality of high-mass IMF (Kroupa 2002). Similar values of the IMF slopes were derived for OB associations in the Milky Way and the Magellanic Clouds (Massey 2003), i.e. in the metallicity range $0.008 \lesssim Z \lesssim 0.02$. Thus our result confirms recent evidence that metallicity does not affect the stellar IMF.

Haiman et al. (1994) obtained steeper average slope for 174 associations in M 31 ( $\Gamma=-2.06 \pm 0.23)$, using the $B V R I$ catalog of Mag92. As seen in Fig. 7, it is inconsistent with our estimates for the lower mass bins, based on HST (OB stars) and 2MASS (RSGs) photometry. Taking into account the variety in stellar density and probably in star formation histories between OB78 and OB79/OB82 (cf. Veltchev et al. 1999), we have sound reasons to believe that the obtained values of $\log \xi$ for these areas are representative for most associations in our sample. We contribute the result of Haiman et al. (1994) to underestimated extinctions towards individual stars, that would reduce the stellar luminosities, and the derived masses. The infrared $J H K_{\mathrm{s}}+I$ photometry of RSGs allowed us to detect more obscured sightlines within the associations and to reconstruct correctly the upper end of the MS strip.

Certainly, our result for high-mass IMF needs to be complemented with studies of less massive populations in M31. A promising opportunity to achieve this goal is the deep UBVRI survey of the Andromeda galaxy, a part of the Local Group Survey (see Massey et al. 2001). We caution, however, that employment of photometry alone for derivation of IMF is not enough effective, and recognize the need of spectroscopy.

The average value of the normalized UV extinction $\left\langle R_{2490}\right\rangle=8.6$ over the associations implies sightlines with $R_{V}=5.5$ (Fitzpatrick 1999), typical for the dense molecular component of the interstellar medium. This finding is in accordance with the result of Bianchi et al. (1996) who emphasized a low possibility for a weaker $2175 \AA$ bump in M 31 extinction curve toward young blue stars in associations, infering $R_{V}>4$ as well. On the other hand, one has to point out the limited applicability of $R_{2490}$ value as an extinction law $\left(R_{V}\right)$ indicator. For example, the analytical relationships of Cardelli et al. (1989) predict a range of possible $R_{2490}$ that is narrower $\left(7.1 \leq R_{2490} \leq 8.2\right)$ and less sensitive to the parameter $R_{V}$ in its conservative limits between 2.6 and 5.9. However, the interpolation between the observational points (see Fig. 1a in Cardelli et al. 1989) at $\lambda \lambda 2175,2800 \AA$ gives $7.6 \pm 0.3 \leq R_{2490} \leq 8.5 \pm 0.7$.

Acknowledgements. This publication makes use of data products from the Two Micron All Sky Survey, which is a joint project of the
University of Massachusetts and the Infrared Processing and Analysis Center/California Institute of Technology, funded by the National Aeronautics and Space Administration and the National Science Foundation. Our work is supported by the grant F1302/2003 of the Bulgarian NSF.

We are grateful to Dr. B. Williams for the kindly provided photometric data, to Dr. V. Ivanov for the valueable discussions and to our referee Dr. E. Magnier for the stimulating comments and suggestions for improving this paper.

\section{References}

Anders, E., \& Grevesse, N. 1989, GeCoA, 53, 197

Bianchi, L., Clayton, G. C., Bohlin, R. C., Hutchings, J. B., \& Massey, P. 1996, ApJ, 471, 203

Bressan, A., Fagotto, F., Bertelli, G., \& Chiosi, C. 1993, A\&AS, 100, 647

Burstein, D., \& Heiles, C. 1984, ApJS, 54, 33

Cardelli, J., Clayton, G., \& Mathis, J. 1989, ApJ, 345, 245

Fagotto, F., Bressan, A., Bertelli, G., \& Chiosi, C. 1994, A\&AS, 104, 365

Fitzpartick, E. L. 1999, PASP, 111, 63

Galarza, V., Walterbos, R., \& Braun, R. 1999, AJ, 118, 2775

Haiman, Z., Magnier, E., Lewin, W., et al. 1994a, A\&A, 286, 725

Haiman, Z., Magnier, E., Battinelli, P., et al. 1994b, A\&A, 290, 371

Hill, J., Isensee, J., Bohlin, R., et al. 1995, ApJS, 98, 595 (H95)

Hunter, D., Baum, W., O’Neil, Jr. E., \& Lynds, R. 1996, ApJ, 468, 633

Hurley, J., Pols, O., \& Tout, C. 2000, MNRAS, 315, 543

Kroupa, P. 2002, Science, 295, 82

Kurucz, R. 1992, in The Stellar Populations in Galaxies. ed. B. Barbuy, \& A. Renzini (Dordrecht: Kluwer), 225

Magnier, E., Lewin, W., van Paradijs, J., et al. 1992, A\&AS, 96, 379 (Mag92)

Magnier, E., Hodge, P., Battinelli, P., Lewin, W., \& van Paradijs, J. 1997, MNRAS, 292, 490

Massey, P. 2002, ApJS, 141, 81

Massey, P. 2003, ARA\&A, 41, 15

Massey, P., \& Johnson, O. 1998, ApJ, 505, 793

Massey, P., Lang, C., DeGioia-Eastwood, K., \& Garmany, C. 1995, ApJ, 438, 188

Massey, P., Hodge, P., Holmes, S., et al. 2001, BAAS, 33, 1496

Mochejska, B., Kaluzny, J., Stanek, K., \& Sasselov, D. D. 2001, AJ, 122,1383

Odewahn, S. 1987, AJ, 93, 310

Pagani, L., Lequeux, J., Cesarsky, D., et al. 1999, A\&A, 351, 447

Salpeter, E. 1955, ApJ, 121, 161

Schaller, G., Schaerer, D., Meynet, G., \& Maeder, A. 1992, A\&AS, 96, 269

Stanek, K., \& Garnavich, P. 1998, ApJ, 503, 131

van den Bergh, S. 1964, ApJS, 9, 65

Veltchev, T., Nedialkov, P., \& Ivanov, G. 1999, Rev. Mex. Astron. Astrofis., 35, 13

Williams, B. 2002, MNRAS, 331, 293

Williams, B. 2003, MNRAS, 340, 143

Williams, B., \& Hodge, P. 2001, ApJ, 548, 190 